\title{
CALIDAD DE VIDA Y CAMBIO SOCIAL. De la polarización social a la axiológica en la sociedad andaluza ${ }^{1}$
}

\author{
CLEMENTE J. NAVARRO YÁÑEZ \\ MANUEL PÉREZ YRUELA \\ Universidad Pablo de Olavide \\ IESA de Andalucía \\ PALABRAS CLAVE ADICIONALES \\ ADDITIONAL KEYWORDS \\ Cultura política, Indicadores sociales, Desigualdad. \\ Political Culture, Social Indicators, Inequality.
}

RESUMEN. En este artículo se presentan los resultados de una investigación sobre condiciones de calidad de vida en la sociedad andaluza, basada en una encuesta realizada a cerca de 2.400 sujetos. La investigación se inscribe en la tendencia reciente a analizar los cambios objetivos en las condiciones de vida de los ciudadanos y, a la vez, analizar la opinión que estos cambios les merecen. A partir de esta comparación entre dimensiones objetivas y subjetivas, se ha realizado una tiplogía para clasificar a la población estudiada según el grado de coincidencia entre ambas dimensiones. El trabajo se ha centrado sobre todo en el tipo "adaptación", en el que se incluyen aquellos sujetos que pese a tener condiciones objetivas por debajo de la media expresan satisfacción subjetiva con su situación. Este es el grupo mayoritario (42\%). En relación a este grupo, en el trabajo se desarrolla una explicación de esta paradoja. Esta se basa en la hipótesis de que los cambios ocurridos en las últimas décadas en las condiciones objetivas de vida, debido sobre todo a la expansión del Estado del Bienestar, han producido un percepción positiva del cambio social. La paradoja de la satisfacción se explicaría por la expectativa de que las condiciones objetivas mejoren a corto plazo dada la tendencia positiva de los cambios recientes experimentados.

\footnotetext{
1 Este trabajo es una versión muy ampliada de otro publicado por los mismos autores: NavarroYáñez, C. y M. Pérez Yruela (1999), "Calidad de vida y cambio social”, en Moyano, E. y M. Pérez Yruela (coord.), Informe Social de Andalucía (1977-1997). Dos décadas de cambio social, pp. 491- 498 .
}

\section{Revista Internacional de Sociología (RIS)}

Tercera Época, n² 26, Mayo-Agosto, 2000, pp. 5-38. 
El estudio sociológico del cambio en las condiciones y calidad de vida de sociedades y grupos humanos se ha venido realizando tradicionalmente a través de indicadores descriptivos, principalmente de carácter cuantitativo. Era menos usual analizar estos cambios teniendo en cuenta la valoración que pudieran hacer de ellos los propios ciudadanos que al fin y al cabo los viven. En otras palabras, el énfasis solía ser menor en las dimensiones subjetivas del cambio y, cuando se utilizaba, se hacía sin relacionar una y otra forma de análisis. No obstante, hoy es cada vez más frecuente emplear ambas perspectivas al mismo tiempo, para comprender mejor las relaciones entre el cambio en las condiciones de vida y la percepción social que existe de ello, pues sólo así se puede aprehender el significado que tienen esos procesos de cambio.

En este artículo se va a seguir esta tendencia más reciente. Se van a analizar cambios objetivos en las condiciones de vida de los ciudadanos y a la vez se van a analizar las opiniones que estos cambios les merecen. A partir de este doble análisis, el artículo se centra en la consideración de la medida en que las dimensiones objetiva y subjetiva del cambio coinciden o no, para, a continuación, intentar explicar las razones de tales coincidencias o divergencias. Estas explicaciones se basan, a su vez, en algunas hipótesis ya sugeridas en otros estudios similares, construidas a partir de la percepción del cambio social por parte de los ciudadanos.

El estudio empírico se ha realizado para el caso de la población andaluza, mediante una encuesta específica sobre "Calidad de Vida en Andalucía (ECVA)", realizada por el Instituto de Estudio Sociales de Andalucía. El trabajo se presenta en cuatro partes. En la primera se hace una revisión de las aportaciones que se han utilizado para la medición de la calidad de vida y para establecer tipologías en torno a ello utilizando al mismo tiempo las perspectivas objetiva y subjetiva. En la segunda, se hace la aplicación al caso andaluz. En la tercera, se discuten las convergencias y divergencias entre las dimensiones objetivas y subjetivas, a partir de la percepción del cambio social. En la última se discuten los resultados de la investigación y se proponen algunas hipótesis para interpretarlos.

\section{MEDICIÓN Y TIPOLOGÍAS}

En los estudios empíricos sobre calidad de vida, este concepto se ha venido midiendo en términos objetivos o subjetivos. En el primer caso, la calidad de vida se mide a través de indicadores descriptivos, que dan cuenta del acceso a determinados recursos que permiten el desarrollo de una "vida buena". Por lo general, la selección de estos indicadores se basa en alguna teoría o concepción sobre las necesidades humanas, de forma que sea posible evaluar a través de ellos hasta qué punto están o no satisfechas esas necesidades en un población determinada ${ }^{2}$. Ejemplos de estos indicadores pueden ser el nivel de renta, el tipo 
y equipamiento de la vivienda o el acceso a servicios básicos como los educativos, los sanitarios o los de bienestar social, entre otros. La valoración de estos indicadores, esto es, el calificarlos mejor o peor, es una cuestión de comparación con determinados estándares. Así por ejemplo, y como es bien sabido, la pobreza medida en términos de ingresos se define por comparación con los ingresos medios de una comunidad, en concreto, aquéllas situaciones en las que los ingresos de una persona son inferiores a la mitad de los ingresos medios de una persona en la comunidad de referencia (Deleeck y Van den Bosch, 1990).

En el segundo caso, la calidad de vida se mide a través de indicadores evaluativos, que dan cuenta de la satisfacción de los individuos con sus condiciones de vida. Estos indicadores también se valoran por comparación, pues reflejan la diferencia existente entre el nivel de aspiraciones y la situación real tal y como es percibida subjetivamente. De hecho, se ha constatado que para evaluar la distancia entre aspiraciones y situación real los sujetos utilizan varios estándares de comparación, como pueden ser la situación en el pasado, la comparación con grupos de iguales o las perspectivas y expectativas sobre el futuro (Campbell et al., 1976; Andrews y White, 1976, Allart, 1978; Michalos, 1980).

Estudios realizados en áreas geográficas con distinto nivel de desarrollo han permitido comprobar que las dimensiones objetiva y subjetiva de la calidad de vida no suelen coincidir. Por ello, se ha insistido en la necesidad de estudiarlas conjuntamente (Allart, 1978; Campbell et al., 1976; Barnes y Kaase, 1979; Fletcher y Lorenz, 1985). La aceptación de este enfoque en la medición de la calidad de vida, que suele denominarse "modelo Michigan" (Michallos, 1980), ha hecho que la investigación se centre cada vez más en la comparación entre indicadores descriptivos u objetivos y evaluativos o subjetivos. Dentro de ello se ha prestado una atención especial a los estándares de comparación que utilizan los sujetos para valorar su situación actual, ya que ello permite explicar de forma adecuada la causa de las diferencias entre expectativas y realidad, tanto en aspectos concretos, como en la calidad de vida en su conjunto concebida como agregación de los distintos aspectos que forman parte de ella.

De acuerdo con lo anterior, se ha definido una tipología en la que puede clasificarse cualquier población en función de los valores que pueden tomar las dimensiones descriptiva y evaluativa. En concreto, se han delimitado cuatro grandes tipos, que se indican en la tabla 1.

\footnotetext{
${ }^{2}$ Estos indicadores se definirían a partir de una teoría sustantiva sobre la buena vida, conjunto de necesidades que deben ser satisfechas para que pueda hablarse de una situación objetivamente satisfactoria. Una teoría de este tipo, con su operacionalización a través de indicadores concretos, puede consultarse en Doyal y Gough (1991).
} 
Tabla 1.

Tipología de la calidad de vida

\begin{tabular}{|l|l|c|c|}
\cline { 2 - 4 } \multicolumn{2}{c|}{} & \multicolumn{2}{c|}{ INDICADORES DESCRIPTIVOS } \\
\cline { 3 - 4 } & \multicolumn{1}{c|}{ Positivo } & Negativo \\
\hline \multirow{2}{*}{$\begin{array}{l}\text { INDICADORES } \\
\text { EVALUATIVOS }\end{array}$} & Positivo & BIENESTAR & $\begin{array}{c}\text { DISONANCIA } \\
\text { Paradoja de la insatisfacción }\end{array}$ \\
\cline { 2 - 4 } & Negativo & $\begin{array}{r}\text { ADAPTACIÓN } \\
\text { Paradoja de la satisfacción }\end{array}$ & PRIVACIÓN \\
\hline
\end{tabular}

Fuente: Adaptado de Olson y Schober (1993).

El tipo "bienestar" recoge los casos en los que básicamente coincide un valor positivo de ambos conjuntos de indicadores. El tipo "privación" recoge los casos en los que también coinciden los valores, en este caso negativos, de los indicadores. En estos dos tipos no existen diferencias apreciables entre los indicadores de las dos dimensiones, por lo que no se plantea problema alguno de explicación de las diferencias. Los tipos "disonancia" y "adaptación" si presentan diferencias, paradojas, que hay que explicar. En el primer caso por que las evaluaciones subjetivas son inferiores a las situaciones objetivas, y en el segundo porque son superiores. Además, en el caso de Andalucía, como se verá más adelante, el tipo "adaptación" recoge un número considerable de casos.

En general, dado que la desigualdad es un fenómeno bastante universal, es previsible encontrar en muchas comunidades una parte más o menos importante de la población cuyas condiciones objetivas sean inferiores a la media de esa misma comunidad y, por supuesto, inferiores a otras de mayor nivel de desarrollo. Por ello, puede esperarse hipotéticamente que estas personas coincidan con los tipos "adaptación" o "privación". En el caso de la adaptación, la explicación de por qué las personas se sitúan en este tipo tiene, como decimos, un interés particular.

Por una parte, interesa encontrar las razones por las que las situaciones de carencia relativa no conducen automáticamente a la situación de "privación", esto es, a una evaluación también negativa de las condiciones de vida en términos subjetivos. En definitiva, interesa conocer que factores intervienen en la evaluación que hacen que pese a las carencias los individuos estén insatisfechos con su calidad de vida.

Por otra parte, interesa conocer estas razones para analizar las consecuencias que pueden derivarse de la situación de "adaptación" para la sociedad en la que 
se produce. La adaptación puede reflejar en unos casos cambios acelerados de mejora de la situación objetiva, que hace que las personas sean optimistas frente al futuro, pues creen que esos cambios llegarán a beneficiarles a corto o medio plazo. Podría decirse que en estos casos la adaptación es un período de espera hasta llegar a la situación de bienestar. En otros casos, sin embargo, la adaptación puede tener un significado social muy distinto. Está bien documentado que formas de vida caracterizadas por situaciones en las que objetivamente se detectan carencias y necesidades junto a índices altos de satisfacción, pueden desembocar en sistemas normativos orientados a la perpetuación de las situaciones de necesidad e incluso de la pobreza y la exclusión social. Esto se debe a un proceso adaptativo por el que las diferencias entre situación real y aspiraciones se reducen progresivamente, pero más por el lado de las segundas, que por el desarrollo de iniciativas por las que tratar de superar la situación de privación.

En ello también pueden influir las agencias provisoras de bienestar, tanto públicas como voluntarias, cuando proporcionan recursos, sobre todo a través de programas asistencialistas tradicionales, sin que los beneficiarios se vean estimulados a cambiar substancialmente sus actitudes o comportamientos para insertarse activamente en la sociedad. Se produce así un efecto no querido como es la formación de subculturas caracterizadas por la dependencia frente a agentes externos y orientadas fundamentalmente a las prestaciones sociales, a los resultados del proceso político, sin intentar participar en la solución de los problemas que les afectan? ${ }^{3}$.

En general, para explicar por qué las personas adoptan posiciones adaptativas se suelen aportar dos tipos de respuestas. La primera es de carácter técnico, se refiere a la escasa validez y fiabilidad de los instrumentos utilizados para medir la satisfacción. Si bien, el desarrollo de nuevos instrumentos, tal y como la escala Life 3 o el Índice de Bienestar que utilizaremos en este texto, y sus respectivos tests de validez y fiabilidad, dejan poco campo a esta crítica (Andrews y White, 1976; Andrews y Robinson, 1991).

Las segundas son de carácter substantivo y, a su vez, pueden dividirse en dos grandes tipos: individuales o psicosociales, por una parte, y estructurales o sociológicas, por otra. Entre las primeras, cabe destacar el enfoque de la "minimización de disonancia cognitiva" de Festinger (1978) y el del "aprendizaje de impotencia" (learned helplessness) propuesto, entre otros, por Seligman (1975) y Abramson (1978). Ambos han sido integrados en un sólo marco analí-

\footnotetext{
${ }^{3}$ Esta sería la denominada cultura política de "súbdito" en la clásica tipología formulada por Almond y Verba (1963), que continúa siendo la obra de referencia clásica sobre cultura política. Sobre la relación entre welfare programı y el concepto de ciudadanía se remite a Kymblica y Norman (1994).
} 
tico en el trabajo de Olson y Schober (1993). Estos autores señalan que, por lo común, las personas prefieren reducir la disonancia cognitiva existente entre su situación objetiva y sus aspiraciones, en vez de asumir los costes que supondría cambiar la situación. Para ello rebajan el nivel de sus aspiraciones o cambian los estándares de comparación. Pero la paradoja de la satisfacción se produce, sobre todo, cuando estas personas perciben que tales costes son muy altos, pues creen, objetiva o subjetivamente, que el cambio de la situación es independiente de su actuación, está fuera de su control, se sienten impotentes. El resultado, según los autores, es la adaptación por resignación ${ }^{4}$.

En los enfoques socioestructurales se atiende, en cambio, a los factores de cambio social. Ipsen (1998), por ejemplo, señala que la adaptación es resultado de la presión social causada por el desarrollo del capitalismo. Ser una persona insatisfecha en una sociedad con niveles materiales de vida medio o altos y oportunidades para poder alcanzarlos puEde llegar a considerarse como una incapacidad personal para progresar en ese contexto, aunque ello pueda deberse a cuestiones completamente ajenas a las características del sujeto. Por esto, la expresión de la insatisfacción tiende a reducirse. A ello añade rasgos del enfoque psicosocial. Dado que el proceso de movilización ligado al desarrollo capitalista implica que cada vez más esferas sociales quedan fuera del control personal, cualquier mejora en la situación personal, por pequeña que sea, es considerada por la persona un logro, una mejora, lo que aumenta la satisfacción personal cuando tales mejoras se producen.

En otros enfoques socioestructurales se añade una perspectiva histórica y culturalista atenta a los cambios sociales. Tal es la posición que sostiene Ronald Inglehart (1991). Apoyándose en una amplia literatura, modelo Michigan, y análisis empíricos sobre decenas de países a través de encuestas, concluye que las variaciones en la satisfacción dependen, fundamentalmente, de dos factores: uno temporal y otro cultural. El primero señala que cambios de condiciones de vida a corto plazo producen aumentos substanciales de satisfacción, mientras que cambios a largo plazo, no implican diferencias importantes. De aquí deriva la "paradoja de la pequeña variación intergrupal". Con esta expresión quiere reflejar el hecho de que las variables que habitualmente explican diferencias en las actitudes, no dan cuenta de las diferencias en la satisfacción con la propia vida. En otras palabras, los grupos que se diferencien por rasgos estables, como por ejemplo el género o el nivel de educación, presentan menos diferencias en la satisfacción con la propia vida que los grupos que se diferencian por rasgos que pueden

\footnotetext{
+ Además de los citados, sobre la importancia del sentimiento de impotencia o alineación sobre la calidad de vida pueden consultarse Yetim (1993), Martinson et cl. (1984) o Abbey y Andrews (1985).
} 
cambiar de manera apreciable a corto y medio plazo, como por ejemplo el nivel de ingresos o el estado civil.

Por otra parte, comprueba que el ajuste cuantitativo entre situación y aspiraciones - la diferencia que da cuenta de la satisfacción o insatisfacción - se produce más rápidamente que el ajuste cualitativo, pues éste último supone cambiar las metas que se persiguen. De esto se deriva que de persistir determinadas condiciones por largo tiempo, incluso después de una mejora objetiva, pueda producirse un aumento de la insatisfacción - una nueva diferencia-, y sobre todo, cambios substanciales en el orden de las preferencias o metas sociales como consecuencia del reemplazamiento generacional.

También se refiere Inglehart a la "paradoja de la gran variación transnacional", en la que se apoya para revelar la importancia de los factores culturales en la satisfacción. Existen patrones culturales específicos que delimitan normas concretas para juzgar sobre la maldad o bondad de la sociedad y la vida en común, sobre la satisfacción o la insatisfacción, pues "las culturas difieren en la medida en que permiten la expresión de la infelicidad e insatisfacción con la propia vida" (Inglehart, 1991: 261). No obstante, los cambios en el nivel de desarrollo económico pueden modificar la influencia de la dimensión cultural. Por ejemplo, una mejora de orden socioeconómico, o lo que es lo mismo, un acercamiento de las condiciones de vida a las aspiraciones, podría dar lugar a mayores índices de satisfacción y felicidad; y viceversa, un aumento de la distancia daría lugar al efecto contrario. Pese a ello, Inglehart ha podido demostrar la existencia de pautas culturales estables para diez países europeos. La pertenencia a esas comunidades culturales, expresada en términos de nacionalidad, explica mejor la satisfacción -más del doble de la varianza- que cualquier otra variable, como nivel de ingresos, género, edad o clase, y que los cambios de tipo económico que pueden producirse en la tasa de inflacción, de crecimiento económico u otros (Ibidem: p. 259).

En suma, Inglehart señala que el cambio en la situación objetiva explicaría las diferencias en satisfacción, sobre todo cuando este cambio es rápido, aunque en todo caso éstas diferencias están moduladas por ciertas pautas culturales de cada sociedad. Además, sin restar importancia a los cambios a corto plazo, el mantenimiento de la mejora por un largo plazo puede dar como resultado, primero, un ajuste cuantitativo generalizado entre situación y aspiraciones, pero en segundo lugar, un cambio substancial de las aspiraciones y, con ello, una nueva distancia entre ambas. En este sentido, diversas investigaciones sobre la base del "modelo Michigan", han permitido constatar que entre todos los estándares de comparación que utilizan los sujetos para comparar situación objetiva y aspiraciones, uno de los más importante es el que utiliza como referente la mejor situación en el pasado (Michallos, 1980; Chamberlain y Zika, 1992; Horley y Lavery, 1991).

Para realizar una contrastación empírica de este marco analítico, se ha utilizado la encuesta citada antes, que se diseñó sobre la base de ese marco de análi- 
sis. En consecuencia, el cuestionario ha incluido preguntas para medir la calidad de vida en términos subjetivos, tanto de carácter general como es la escala Life3 o el Indice de Bienestar, como de carácter específico. Se han incluido preguntas sobre evaluaciones subjetivas de aspectos como la vivienda y sus equipamientos, los equipamientos urbanos de la zona de residencia, las políticas públicas en general y las de bienestar social en particular, o las expectativas futuras de mejora personal, entre otras. También se han incluido preguntas sobre valoración subjetiva de la evolución de algunos de los aspectos anteriores en los últimos años, para medir la percepción del cambio social. Finalmente, se han incluido preguntas de carácter objetivo sobre aspectos específicos como pueden ser de nuevo la vivienda, las percepciones recibidas a través del sistema de bienestar social, los ingresos familiares o la posición social de los entrevistados, entre otras 5 .

\section{EVALUACIÓN SUBJETIVA Y TIPOLOGÍAS EN ANDALUCÍA}

De acuerdo con los datos obtenidos en la ECVA, la percepción subjetiva que tienen los andaluces sobre su calidad de vida permite calificar a la sociedad andaluza como una sociedad satisfecha. Al menos, así lo reflejan los indicadores. Medida la satisfacción con la vida en su conjunto a través de la escala Life3, alrededor del $70 \%$ de los 2.300 entrevistados se declaran satisfechos. Propuesta la misma escala de siete puntos para 17 áreas distintas — familia, actividades de ocio, trabajo, medio ambiente y otras-, resulta una media cercana al 5. Además, el Indice de Bienestar, construido a través de una escala de afectos y otra de satisfacción, presenta una media cercana a 9 , dos puntos superior a su punto medio ${ }^{6}$.

Estos altos niveles de satisfacción hay que ponerlos en relación con las características de la sociedad andaluza, que objetivamente, y pese a los avances en todos los sentidos de las dos últimas décadas, tiene rasgos todavía con valores inferiores a la media española? ${ }^{7}$. Además, una sociedad en la que persisten pro-

\footnotetext{
${ }^{5}$ Sobre aspectos metodológicos y técnicos de la ECVA puede consultarse Fernández Esquinas, M., C. Gutierrez y M. Trujillo (1998).

"La evaluación que los propios sujetos hacen de su calidad de vida contiene tanto elementos cognitivos - percepción de la situación objetiva- como afectivos. Respecto a los primeros, se ha constatado que la Escala de Satisfacción (SWLS) muestran los mejores niveles de validez y fiabilidad (Larsen et al., 1985). En cambio, la escala Life3 y el Indicador de Bienestar General se han mostrado más valiosas y fiables para medir los dos aspectos de forma conjunta (Andrews y White, 1976; Andrews y Robinson, 1991). Al ser la calidad de vida un fenómeno multidimensional, también se ha replicado la escala Life3 para diversas áreas, con lo que se garantiza también la validez y fiabilidad de las mediciones (Diener, 1994: 146-147). Para escoger las áreas objeto de las preguntas se han seguido las indicaciones de Andrews y White (1976).
} 
blemas y desigualdades sociales como pueden ser los niveles de rentas de las familias, las tasas de pobreza o las de desempleo, algunos de ellos con más gravedad que en otras Comunidades Autónomas. A la vista de estos datos parece que el tipo "adaptación" debe tener en Andalucía un peso especial. Efectivamente así se confirma cuando se analizan las tipologías de calidad de vida en Andalucía.

Para analizar las tipologías se ha procedido de la siguiente forma. Sobre los datos obtenidos en la encuesta se ha creado un conjunto de variables nuevas e indicadores sintéticos, también de carácter descriptivo y evaluativo, apropiados para analizar los cuatro tipos de calidad de vida descritos. La tipología se ha basado en cuatro áreas concretas sobre las que había suficiente información en el cuestionario: posición social del entrevistado, posición social de la familia del entrevistado, vivienda y situación económica (ingresos/pobreza). La descripción de los indicadores descriptivos y evaluativos se presenta en la tabla 2. Cada uno de ellos corresponde a una pregunta de la encuesta o a varias preguntas sobre el mismo tema, cuyas respuestas se han integrado en un indicador sintético para cada área ${ }^{8}$

Una vez determinados y cuantificados los cuatro tipos de calidad de vida en cada área, se ha aplicado análisis de correspondencias múltiples (ACM) para determinar si existe relación entre ellos, esto es, entre las áreas. Posteriormente se realiza un análisis de cluster con las puntuaciones factoriales de cada entrevistado en las dimensiones resultantes y se aplican tests de homogeneidad de varianzas ( $\mathrm{T}$ de Tukey y $\mathrm{F}$ de Snedecor) para determinar la validez del agrupamiento".

\footnotetext{
${ }^{7}$ Para ver la evolución de la sociedad andaluza en los últimos veinte años, así como la evolución de aspectos tales como la educación, sanidad, pobreza, o desigualdad social, entre otros temas, y su comparación con la media española puede consultarse Moyano, E. y M. Pérez Yruela (1999).

${ }^{*}$ Se han tomado estas cuatro áreas por tres razones: dan cuenta de las condiciones mínimas de vida, son susceptibles de medición para toda la población entrevistada y existen en el cuestionario indicadores descriptivos y evaluativos para cada una de ellas. Los indicadores que se muestran en la tabla son los que se han mostrado más significativos en análisis previos, pues en origen se han utilizado todos los que existían en la encuesta. Por otra parte, para determinar su fiabilidad y validez se han utilizado distintos métodos, en función del tipo de indicador: test alpha de Cronbach cuando el indicador es resultado de un sumatorio, análisis factorial de correspondencias múltiples (ACM) o análisis de componentes principales no lineal ( $\mathrm{ACPnl}$ ), cuando se trata de crear dimensiones que resuman información. Por último, el criterio para determinar situaciones favorables o desfavorables en cada indicador ha sido la media de la distribución o de la escala.

" Este es el procedimiento seguido en cada una de los análisis que se presentan en el texto.
} 
Tabla 2.

Indicadores descriptivos y evaluativos para medir la calidad de vida a partir de la ECVA.

\begin{tabular}{|c|c|c|}
\hline AREA & $\begin{array}{l}\text { INDICADORES } \\
\text { DESCRIPTIVOS }\end{array}$ & $\begin{array}{l}\text { INDICADORES } \\
\text { EVALUATIVOS }\end{array}$ \\
\hline $\begin{array}{l}\text { 1. POSICIÓN SOCIAL } \\
\text { FAMILIAR }\end{array}$ & $\begin{array}{l}\text { E7, E14, E16: Clase social } \\
\text { cabeza familia. } \\
\text { E10 y E11: Ingresos medios } \\
\text { mensuales por persona. } \\
\text { A3: Equipamientos hogar. }\end{array}$ & $\begin{array}{l}\text { J4: Familia. } \\
\text { J9: Actividades familiares. }\end{array}$ \\
\hline 2. RENTA/POBREZA & $\begin{array}{l}\text { El0 y Ell: Renta media } \\
\text { equivalente mensual } \\
\text { por persona. }\end{array}$ & $\begin{array}{l}\text { E13: Percepción situación } \\
\text { socioeconómica. } \\
\text { J5: Dinero del que dispone. } \\
\text { J14: Capacidad de compra. }\end{array}$ \\
\hline 3. VIVIENDA & $\begin{array}{l}\text { A9: Régimen de propiedad. } \\
\text { A5: Baño. } \\
\text { A11: Razones para alquilar. }\end{array}$ & $\begin{array}{l}\text { J8: Vivienda. } \\
\text { A12: Vivienda. }\end{array}$ \\
\hline $\begin{array}{l}\text { 4. POSICIÓN SOCIAL } \\
\text { DEL ENTREVISTADO }\end{array}$ & $\begin{array}{l}\text { E3: Género. } \\
\text { E6: Edad. } \\
\text { E8: Nivel de estudios. } \\
\text { E7: Situación laboral. }\end{array}$ & I3: Posición social subjetiva. \\
\hline
\end{tabular}

Se señalan las preguntas que aparecen en el cuestionario.

Los resultados para cada una de las áreas analizadas muestran que los grupos más importantes son los tipos bienestar y adaptación, alrededor del $60 \%$, seguidos de la privación, entre el 20-25\%, y la disonancia, alrededor del 10\%. Además, los resultados del análisis muestran que estas situaciones se encuentran bastante relacionadas para las cuatro áreas, lo que puede apreciarse en la ilustración 1, donde aparece el primer plano factorial. Esta muestra un eje, el primero, que diferencia entre bienestar y privación, y otro, el segundo, que diferencia entre adaptación y disonancia. El tercero, que aparece junto al primero en la ilustración 2, permite diferenciar entre disonancia, por un lado, y los otros tipos por el otro ${ }^{10}$.

${ }^{10}$ Las ilustraciones representan planos factoriales, en los que se cruza el primer eje factorial, siempre en horizontal, con el segundo o tercer ejes, siempre en vertical. La localización espacial de las categorías muestra sus saturaciones con respecto a los ejes representados en la ilustración. 
Ilustración 1.

Tipología de calidad de vida: situación económica, vivienda, posición social y posición familiar. Saturaciones de las categorías en el primer plano factorial $(50,79 \%$ varianza)

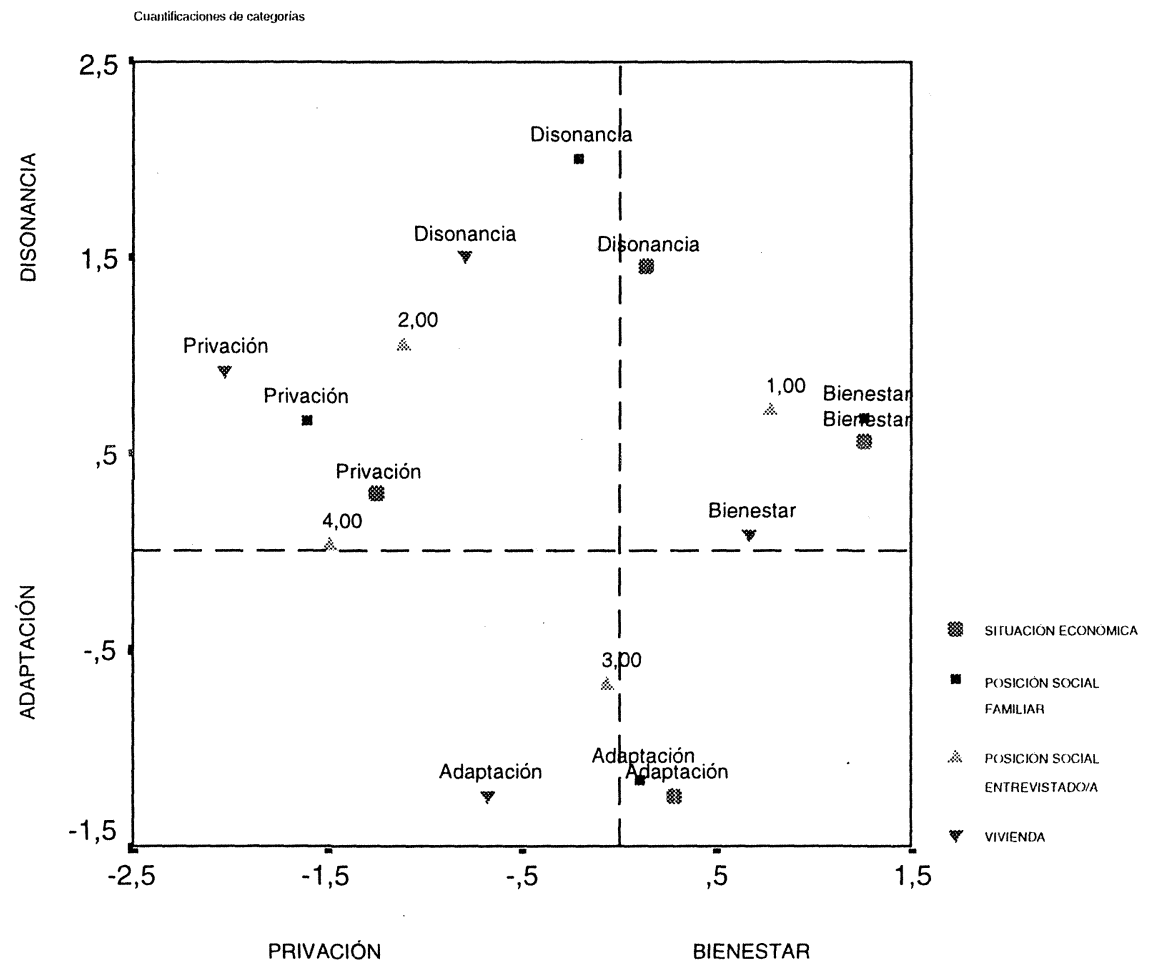

Agrupadas las puntuaciones factoriales de los tres primeros ejes mediante análisis cluster, resultan cuatro tipos que se corresponden con los cuatro polos de la ilustración 1. Su distribución de frecuencias, en la ilustración 3, muestra que el grupo más numeroso corresponde al tipo "adaptación" $(42,5 \%)$, seguido del tipo "bienestar" $(30,9 \%)$, del tipo "privación" $(15,7 \%)$ y del de "disonancia" $(10,9 \%)$.

Efectivamente se confirma la importancia del tipo "adaptación", que nos enfrenta con la necesidad de explicar la "paradoja de la satisfacción" en Andalucía. Según las explicaciones al uso de esta paradoja, expuestas en el punto anterior, la satisfacción de la sociedad andaluza puede explicarse por la combinación de la intensidad y forma en que ha se ha producido el cambio social en esta comunidad en las últimas décadas. Pero, sobre todo, puede explicarse por la percepción 
Ilustración 2.

Tipología de calidad de vida: situación económica, vivienda, posición social y posición social familiar. Saturaciones de las categorías en el segundo plano factorial $(44,15 \% \%$ varianza $)$.

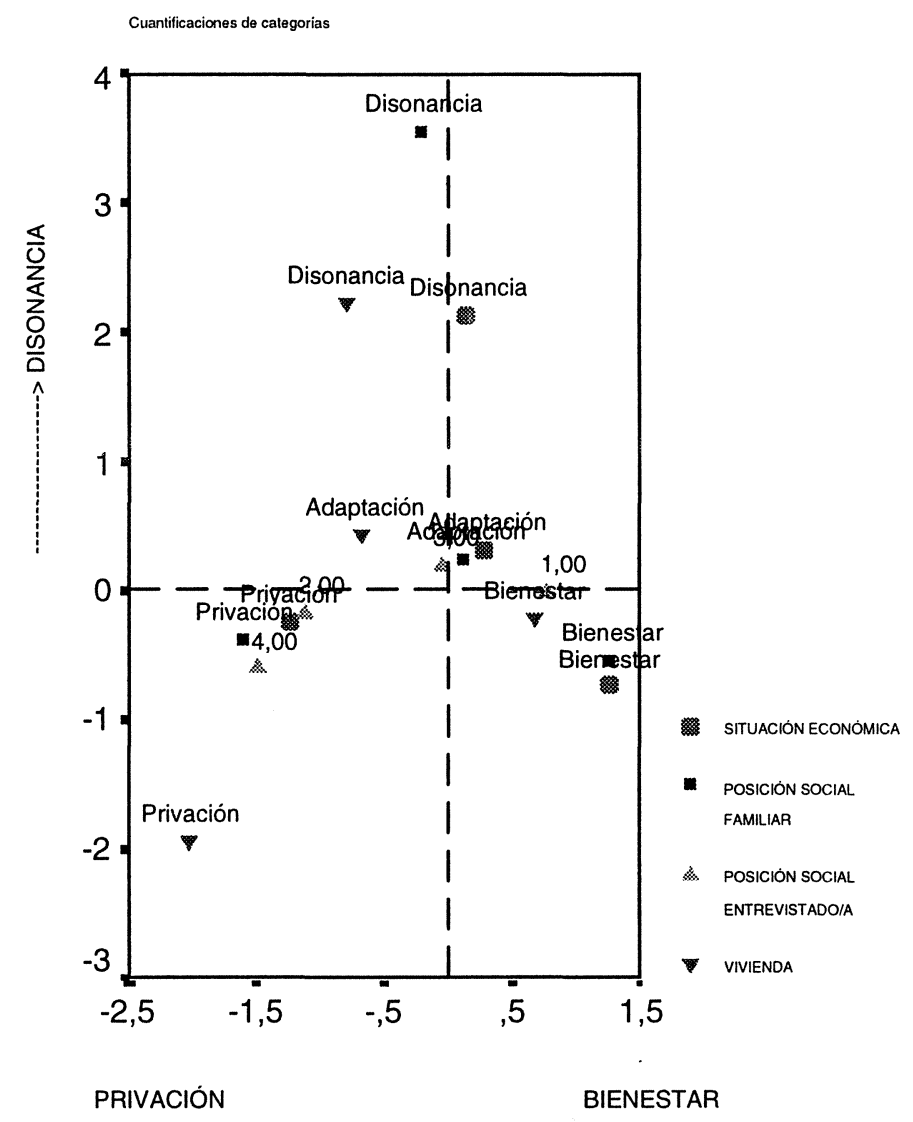

y valoración que tiene la población andaluza de los cambios que ha vivido, por ciertas pautas culturales referidas a la percepción de las carencias y desigualdades, entendidas en sentido amplio, así como por la relación que mantiene la ciudadanía con lo público, con la política. En suma, creemos que la hipótesis principal que puede plantearse para explicar las diferencias identificadas en la percepción de la calidad de vida es que éstas se deben a diferencias entre la percepción del proceso de modernización (mejoras materiales y sobre todo la acción del Estado del Bienestar), y las peculiaridades culturales de la sociedad andaluza, tanto de carácter general, como específicas referidas a la cultura política. 
Tipología de calidad de vida en Andalucía. Importancia relativa de cada tipo sobre el total de la muestra.

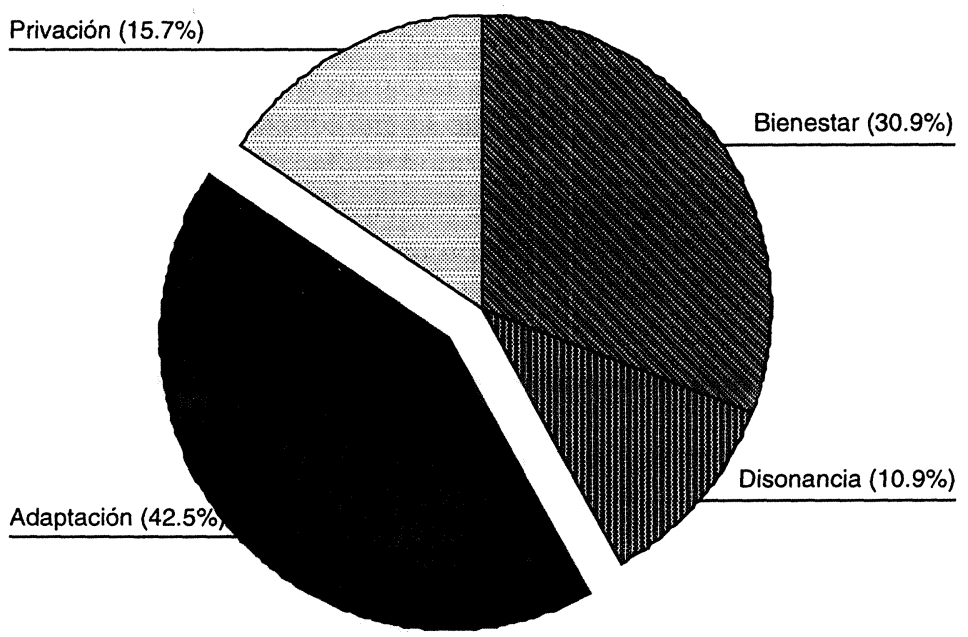

Es indudable que la mejora de las condiciones de vida en Andalucía en el corto periodo de algo más de dos décadas pueden producir el nivel general de satisfacción señalado. En ello ha contado, en gran parte, la extensión del Estado del Bienestar: el acceso a la sanidad, la reducción de los niveles de analfabetismo y el incremento de personas con estudios universitarios, el acceso y la mejora en las condiciones de la vivienda, las políticas de protección social, las relacionadas con los desempleados, o la extensión de los servicios sociales, con especial atención a sectores específicos, como la tercera edad o la infancia, entre otras.

Ahora bien, esta mejora sólo explicaría el aumento en el nivel general de satisfacción, pero no explicaría la existencia de los cuatro tipo de calidad de vida - bienestar, adaptación, disonancia y privación - entre la población andaluza y, particularmente, el caso de la adaptación. Para ello, según lo expuesto en el punto anterior, deberían tenerse en cuenta las diferencias en la percepción del cambio social. En concreto, cabe esperar que cuanto más positiva sea la percepción del cambio social - las experiencias de movilidad social, la reducción de desigualdades o las oportunidades vitales-, con mayor o menor independencia 
de su objetividad, más probable será la aparición del fenómeno de la adaptación entre aquéllos que se encuentran en situaciones objetivas de carencia, pues han percibido un aumento considerable de éstas en un corto periodo de tiempo. Aún presentando condiciones de vida desfavorables, éstas son percibidas como mucho mejores que las existentes en el pasado, habiéndose reducido así la diferencia entre aspiraciones y situación objetiva, lo que da cuenta de la satisfacción.

No obstante, para comprender el cambio social en Andalucía en toda su amplitud y complejidad, así como su relación con la calidad de vida, deben tenerse también en cuenta las normas culturales. La sociedad andaluza presenta en la actualidad una mezcla de rasgos en los que se entrecruzan pautas culturales tradicionales con pautas más propias de la modernidad. El peso de unas y otras varía en función de la edad, los niveles de formación, la ocupación o los lugares de residencia. De manera que no es fácil pronunciarse con carácter general para toda la sociedad andaluza sobre cual de los dos modelos predomina. En cualquier caso, lo que si puede afirmarse es que las pautas tradicionales tienen peso importante en la cultura andaluza, y no son en absoluto una cuestión residual o minoritaria. De forma sucinta, en Andalucía prevalecen formas y actitudes de familismo tradicional, intensa expresión de la religiosidad popular, heteronomía moral, lejanía respecto al mundo público y político, y una actitud que podría calificarse de conformista, por cuanto se hace de la acción estatal, más que de la acción o el logro individual, el centro y motor del dinamismo social y económico (Del Pino y Bericat, 1998). Todo ello en el marco de una red difusa de sociabilidad comunitaria, en la que la familia y las redes de solidaridad juegan un papel muy relevante como sostenedores de situaciones de carencia (Pérez Díaz et al., 1998). En este contexto cultural, las situaciones de carencia relativa, y en su caso de pobreza, se perciben como males sociales, que aun habiéndose reducido en las últimos diez años, atrapan a quienes afectan, al tiempo que refuerzan poco las actitudes para salir de esas situaciones mediante la acción individual (Del Pino y Bericat, 1998: 207).

La conjunción de la mejora en las condiciones de vida, aunque en algunos aspectos sea en niveles inferiores a las de otras regiones, las normas culturales de tipo tradicional, la acción estatal y las redes de ayuda comunitaria, configuran un discurso social y una autopercepción de las situaciones de carencia relativa que hace que puedan sobrellevarse de manera digna y aceptable para quienes las experimentan. En este contexto cultural, sería un mal social que no es fácil eludir, que se comparte abiertamente con los iguales, y para el que no faltan la solidaridad más lejana que llega a través de la política social, y la más cercana que llega a través de las redes amicales y de sociabilidad. A la importancia del cambio social y económico rápido e intenso, se une un marco cultural tradicional, más atento a la acción de los otros para resolver las situaciones de privación que a la acción propia. Por ello, la mejora objetiva en el orden material ha reducido las diferencias respecto a las aspiraciones, pero unido esto a las pautas cul- 
turales tradicionales, ha favorecido, sobre todo, la adaptación de las aspiraciones a la mejoras habidas.

Así pues, sigue habiendo situaciones de carencia relativa, algunas graves, pero con un amplio nivel de integración social, en sentido amplio, y, sobre todo, un nivel de acceso a bienes materiales que veinte o treinta años antes no era imaginable. Se tienen necesidades, pero se cuenta con una red de autoayuda familiar que ayudan a cubrirlas; se tienen carencias, pero se accede a una serie de servicios y prestaciones sociales de carácter público que ayudan a reducirlas. Como se dice coloquialmente, pese a las carencias y necesidades, "no se vive malamente, se vive con dignidad y a gusto" ".

En el marco de estas explicaciones hipotéticas sobre la forma en que los andaluces perciben su calidad de vida, cabe esperar que la situación de privación aparezca ligada a una percepción negativa del cambio social. Esto es, a una percepción negativa de la evolución de las desigualdades y de las situaciones de necesidad y pobreza, a experiencias negativas de movilidad social y a expectativas bajas de mejora a nivel personal o familiar. Cabe esperar una percepción opuesta del cambio social y de las expectativas ligadas a él en el caso de los adaptados, ciudadanos optimistas y esperanzados. Estos, cuando valoran los cambios sociales y las expectativas de futuro, perciben una mejora considerable de su situación personal y familiar, en una tendencia general de reducción de desigualdades. Ahora bien, en ambos casos se trata de grupos de población que, en los indicadores de cultura política que hemos utilizado, manifiestan los que en el lenguaje de la sociología política se denomina incompetencia política: valoran en mucho la acción estatal, pero se declaran incapaces de influir en las decisiones y se consideran poco informados sobre política.

En los ciudadanos en posición de bienestar cabe esperar una percepción del cambio social más neutra, e incluso más negativa, que lo que se de en la situación de adaptación. Su satisfacción estaría determinada simple y llanamente por el ajuste entre situación real y aspiraciones, a través del logro individual más que de la acción de agentes externos. En cambio, los que muestran el dilema de la insatisfacción - la disonancia- cabe esperar que tengan una visión más bien negativa del cambio, pues su insatisfacción mostraría que perciben haber perdido posibilidades de llevar la vida que desearían.

Para completar este marco analítico, debe atenderse a las diferencias en el tamaño de hábitat, pues estas revelan en mayor medida el efecto conjunto del proceso de modernización y de la persistencia de ciertas características cultura-

\footnotetext{
"Como hemos indicado más arriba, la puntuación que se corresponde con el calificativo «a gusto» es el valor modal de la escala Life 3 propuesta a los entrevistados para que evaluasen su calidad de vida.
} 
les. Como es sabido, el urbanismo, tanto en su dimensión física, como en su dimensión social, como forma de vida, es un buen indicador del proceso de modernización (Dickens, 1990). Respecto a la primera dimensión, porque aumenta las oportunidades de acceso de los ciudadanos a las estructuras político-administrativas y a diversos recursos (empleo, renta, estudios, información u otros). Respecto a la segunda, porque incentiva el proceso de individuación característico de la modernidad. De forma esquemática, la ciudad es anonimato, relaciones impersonales guiadas por principios de racionalidad instrumental, desconfianza frente a los otros; el pueblo implica un mayor conocimiento y contacto cotidiano, acciones guiadas por valores y tradiciones, en una red difusa de sociabilidad y ayuda mutua, que aún no dejando de estar presente en la ciudad, es característica del medio rural y procura mayor integración social ${ }^{12}$.

De hecho, la modernización en su dimensión cultural aparece rezagada en los pueblos andaluces con respecto al ámbito urbano. Existe en ellos menor nivel de desconfianza frente a los otros, actitudes laborales que o bien no conceden valor al trabajo o centradas en la valoración del salario, sin atender al prestigio, el logro o la responsabilidad. A ello se une mayor nivel de confianza en la acción de los actores institucionales, y en concreto, en el Estado, que en la sociedad civil. Las situaciones de carencia relativa y de pobreza, aún en los casos en que puedan ser responsabilidad de los propios afectados, tienden a verse como responsabilidad de la actuación pública. Finalmente, en este ámbito, aun con leves diferencias respecto al ámbito urbano, se da mayor grado de religiosidad y de influencia de la religión en la propia vida (Del Pino y Bericat, 1998).

Pese a los todavía débiles procesos de individuación y al mantenimiento de invocaciones externas para la solución de los problemas, bien en el ámbito de las creencias religiosas (Dios) o de la comunidad política (Estado), la dimensión material de la modernización se ha hecho cada vez más presente en los pueblos andaluces. Sobre todo por la acción pública, en el marco general de un continuo proceso de descentralización del Estado de Bienestar (Navarro, 1998). La mejoras en este sentido han sido evidentes en temas como el acercamiento de los servicios sanitarios, educativos, los servicios sociales de base o las prestaciones por desempleo. Esto ha sido más apreciable cuando se compara con la situación en el pasado. Todo ello, junto a las redes de sociabilidad y ayuda mutua, en un marco caracterizado por el sentido de impotencia e incompetencia política, hace previsible que el fenómeno de la adaptación, de la paradoja de la satisfacción, aparezca en mayor medida asociado al ámbito rural, ligada a una visión muy positiva del cambio social, que al ámbito urbano en Andalucía.

\footnotetext{
${ }^{12}$ Sobre el urbanismo como forma de vida moderna, sus rasgos, y sus diferencias respecto al ámbito rural, puede consultarse el clásico Urbanism as a way of life de Louis Wirth.
} 
Los datos de la ECVA permiten hacer algunos análisis adicionales para contrastar hasta qué punto las explicaciones que se han desarrollado en los párrafos anteriores son o no verosímiles. A ello se dedica el punto que sigue.

\section{CAMBIO SOCIAL, ESTADO DE BIENESTAR Y CULTURA POLÍTICA EN ANDALUCÍA}

\section{Percepción del cambio social: una sociedad optimista}

Para poder establecer mediante técnicas estadísticas apropiadas la existencia de asociaciones entre los tipo de calidad de vida y la percepción del cambio social, se han construido los siguientes indicadores, a partir de los datos de la ECVA:

a) Percepción de movilidad social, medida como diferencia entre la autopercepción de posición social (pregunta I5) y la percepción de la posición de la familia de origen (pregunta I6). Los tipos resultantes son: movilidad ascendente (posición social de la familia de origen más baja que la del entrevistado; movilidad descendente (posición social de la familia de origen más alta que la del entrevistado); y no movilidad o herencia (posición social de la familia de origen igual que la del entrevistado).

b) Percepción del cambio en las desigualdades, medida mediante la comparación de la percepción de la evolución de las desigualdades (pregunta I2) y la situación presente (pregunta I1). Los tipos resultantes se muestran en la tabla 3.

c) Percepción de oportunidades vitales, medida como resultado del cruce entre la percepción de las posibilidades de mejorar el nivel de vida del entrevistado y su familia (pregunta I3), con la percepción del entrevistado acerca de las oportunidades que tendrán sus hijos para mejorar (pregunta I4). Los tipos resultantes se muestran en la tabla 4.

Tabla 3.

Percepción del cambio en las desigualdades.

\begin{tabular}{|c|c|c|c|c|}
\hline & \multicolumn{3}{|c|}{ LAS DESIGUALDADES SOCIALES HAN.... } \\
\hline & & Aumentado & Siguen igual & Disminuido \\
\hline \multirow[t]{2}{*}{$\begin{array}{l}\text { EN LA } \\
\text { ACTUALIDAD } \\
\text { EXISTEN... }\end{array}$} & $\begin{array}{l}\text { Grandes desigualdades o } \\
\text { desigualdades importantes }\end{array}$ & $\begin{array}{l}\text { Sociedad en } \\
\text { polarización }\end{array}$ & \multirow[t]{2}{*}{$\begin{array}{l}\text { Sociedad } \\
\text { estática }\end{array}$} & \multirow[t]{2}{*}{$\begin{array}{l}\text { Sociedad } \\
\text { en progreso }\end{array}$} \\
\hline & Pocas desigualdades & Sociedad de Riesgo & & \\
\hline
\end{tabular}


Tabla 4.

Percepción de las oportunidades vitales.

\begin{tabular}{l|lcc}
\multicolumn{2}{c}{} & \multicolumn{2}{c}{$\begin{array}{c}\text { PERCEPCIÓN ACTUAL } \\
\text { OPORTUNIDADES VITALES }\end{array}$} \\
\cline { 3 - 4 } \multicolumn{2}{l|}{} & Positiva & Negativa \\
\hline PERCEPCIÓN OPORTUNIDADES & Positiva & $\begin{array}{c}\text { Optimista } \\
\text { Resignada }\end{array}$ & $\begin{array}{c}\text { Esperanzada } \\
\text { Pesimista }\end{array}$ \\
\hline VITALES PARA LOS HIJOS & Negativa & &
\end{tabular}

d) Valoración de la evolución de la situación social. Indicador sintético construido a partir de la valoración que los entrevistados realizan de diez áreas mediante una escala de cinco puntos al ser preguntados por su opinión acerca del cambio experimentado en cada una de ellas en los últimos diez años (pregunta D2) ${ }^{13}$. Posteriormente el indicador se ha agrupado en tres categorías, tomando como criterio el punto medio de la escala: la situación ha empeorado ("peor"), la situación sigue igual ("igual"), y la situación ha mejorado ("mejor").

El análisis de la visión del cambio social por parte de los ciudadanos andaluces muestra que se trata de una sociedad satisfecha y optimista. A los índices positivos de satisfacción y bienestar aportados al inicio del apartado anterior, debe sumarse que, aunque la mitad de los entrevistados perciben Andalucía como una sociedad estática, en la que según su opinión las desigualdades han cambiado poco desde hace diez años, alrededor del $20 \%$ piensan que se trata de una sociedad en progreso, en la que se han ido reduciendo las desigualdades. Sólo el $2 \%$ cree que aun no habiendo existido desigualdades, existe el riego de que aumenten, mientras que el $24 \%$ cree que aumentarán, habiendo existido anteriormente. En cuanto a la comparación del cambio social habido en la última década y las oportunidades de mejora que los entrevistados perciben para ellos mismos y para la próxima generación, la visión es ampliamente positiva. Según la clasificación de percepción de las oportunidades que se señaló antes (tabla 4), esta percepción es optimista para el $40 \%$ y esperanzada para el $25 \%$. La visión pesimista acumula al $30 \%$ de los entrevistados, mientras que la resignada tan sólo algo más del $3 \%$.

\footnotetext{
${ }^{13}$ Para determinar la validez del indicador sintético se ha realizado un test de fiabilidad mediante el modelo alpha de Cronbach, habiendo dado como resultado un alpha cercano al 0,85 .
} 
Respecto a la evolución de la situación social, sólo el $11 \%$ manifiesta que ha empeorado en los últimos diez años, frente al $29 \%$ que cree se ha mantenido igual, y el $60 \%$ que cree que ha mejorado. Pero esta visión positiva y satisfecha se manifiesta, sobre todo, en la percepción de la movilidad social. Tan sólo el $12,5 \%$ creen haber descendido socialmente, el $42,9 \%$ perciben haberse situado en una posición similar a la de su familia de origen, mientras el $44,6 \%$ restante cree haber descrito una trayectoria ascendente en la escala social en relación a la familia de sus progenitores.

Ahora bien, ¿cómo se asocia este conjunto de percepciones del cambio social en términos de reducción de las desigualdades y percepción de las oportunidades futuras con los tipos de calidad de vida?. En la ilustración 4 se representa esta asociación. Existen tres actitudes básicas: la optimista, en el lado derecho de la ilustración, la pesimista, en el lado izquierdo, y una actitud más neutra en el centro del plano. A ello debe añadirse que, según el segundo eje factorial, la situación de disonancia aparece ligada a una visión neutra, aunque se percibe Andalucía como una sociedad de riesgo; mientras que la situación de privación aparece ligada a la visión más negativa del cambio social (sociedad en polarización, movilidad descendente y visión pesimista). En la tabla 5 se muestra el porcentaje de entrevistado que supone cada grupo, así como las relaciones existentes entre categorías de las variables ${ }^{14}$.

La tabla 5 muestra que la situación de bienestar aparece ligada a una visión estática del cambio en las desigualdades, pues estos ciudadanos no aprecian mejoras substanciales en este aspecto, y aunque expresan una pérdida de estatus social con respecto a la familia de origen, no perciben riesgo de aumento de desigualdades sociales en el futuro. Diferente es la percepción en el tipo "disonancia" que recoge a las personas con buenas condiciones objetivas pero insatisfechas. En este caso, a la pérdida de estatus o, al menos, de no mejora ("no movilidad"), se suma una percepción negativa del cambio habido y de incertidumbre hacia el futuro. La situación de "privación" es la que aparece ligada a una visión más negativa del cambio social. Los entrevistados que se sitúan en este tipo perciben pérdida de estatus y valoran negativamente el cambio en materia de desigualdades sociales. Creen que en la sociedad andaluza han existido y seguirán existiendo grandes desigualdades, y tienden a verla, por tanto, como una sociedad polarizada en términos de clases sociales.

\footnotetext{
${ }^{14}$ Para determinar el peso de cada grupos se ha realizado un análisis cluster sobre las puntuaciones factoriales de los sujetos. En general, las saturaciones de las categorías en cada eje, así como el análisis postfactorial tri-deux realizados, que muestra como se relacionan las categorías entre sí, dan cuenta de la estructura que se refleja en la tabla 5.
} 
Ilustración 4.

Calidad de vida y percepción del cambio social. Saturaciones sobre el primer plano factorial $(49,69 \%$ varianza).

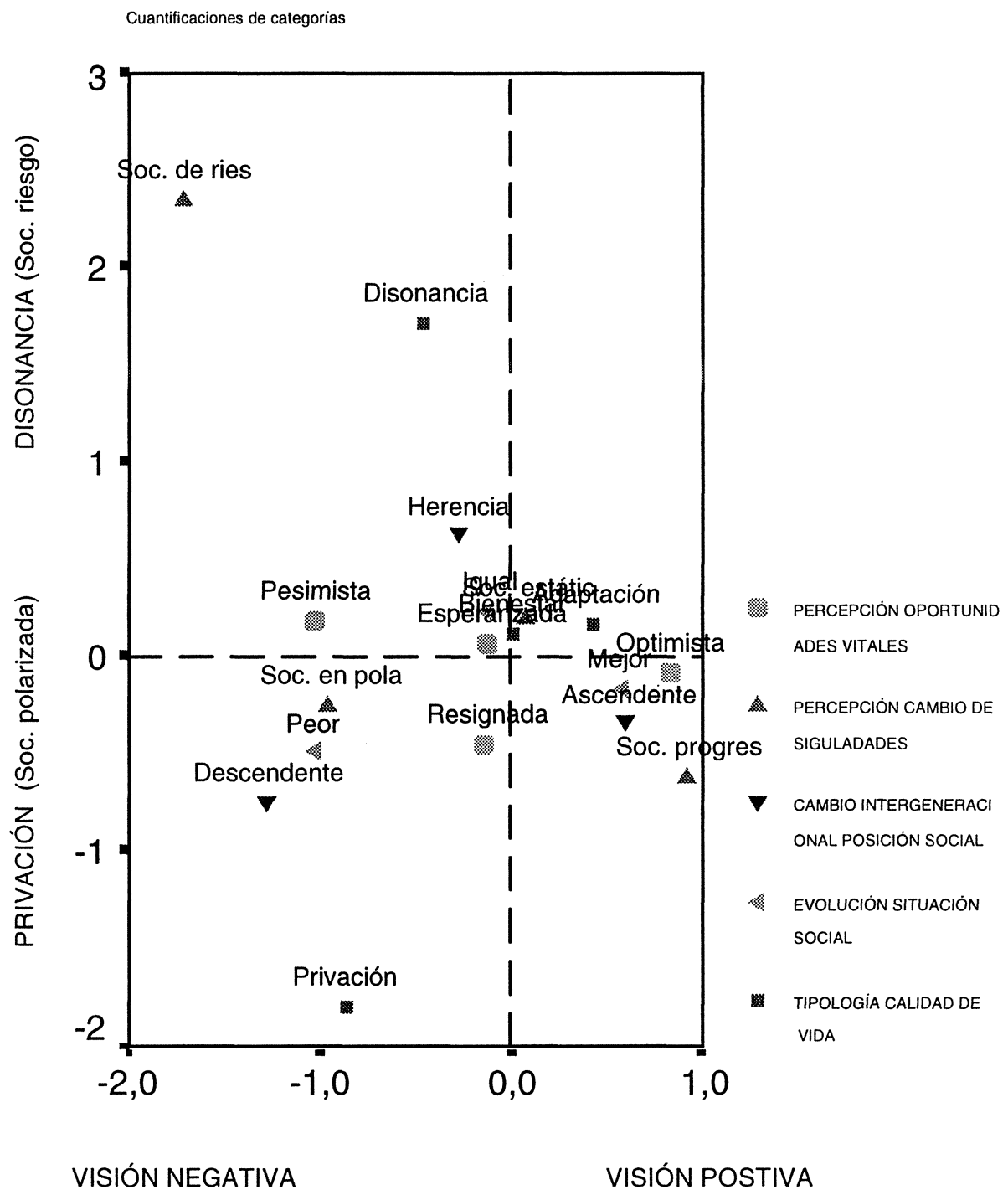


Los entrevistados que se sitúan en el tipo "adaptación" muestran la visión más optimista de la sociedad y del cambio social. Además de ser los únicos que perciben haber aumentado su estatus social con respecto al de sus familias de origen, piensan que han disminuido las desigualdades sociales y que se seguirán reduciendo en el futuro, todo ello en el marco de una mejora general de la situación socioeconómica de Andalucía.

Así pues, parece poder confirmarse que percepción del cambio social y calidad de vida están asociados, y sobre todo, que la adaptación aparece ligada a la visión más optimista del cambio. Ello pese a encontrarse en situaciones objetivas en las que existe algún tipo de carencia en comparación con las condiciones medias de la sociedad. En otros términos, se trata de personas que por su percepción positiva del cambio esperan poder acceder a mejoras reales en sus condiciones objetivas. No se sienten excluidos del proceso general de cambio y modernización de Andalucía, que confían que contribuya a mejorar su situación actual.

\section{Competencia política y Estado del Bienestar}

Para determinar las asociaciones existentes entre los tipos de calidad de vida, la actitud hacia el Estado del Bienestar y la cultura política de los encuestados, se han construido los siguientes indicadores:

a) Indicador de competencia política: construido a través de la autopercepción de competencia cognitiva y capacidad de influencia subjetiva, como indicadores de la capacidad sentida de actuar para transformar las condiciones sociales (pregunta H3). Las categorías de este indicador son las siguientes: competente (se considera influyente e informado), influyente o informado, y no competente (no se considera ni influyente ni informado).

b) Indice de apoyo al estado del bienestar: mide las actitudes, a favor o en contra, sobre las políticas de ayuda a los desfavorecidos (pregunta D5). Este indicador, operacionalizado a través de ocho items con escala de 7 puntos, se ha resumido en dos posiciones: más bien a favor (apoyo, por encima del punto medio) y más bien en contra (no apoyo, por debajo del punto medio) ${ }^{15}$.

c) Sistema de bienestar: mide la prevalencia de los agentes de provisión de bienestar en relación a la familia. Se ha construido aplicando análisis de correspondencias múltiples a las variables de la sección B del cuestionario, dedicada al tema "Integración Social y Servicios Sociales". Los indicadores que se han revelado más apropiados son: la percepción o no por las familias de sociales por

\footnotetext{
${ }^{15}$ También en este caso se ha aplicado el modelo alpha de Cronbach para determinar la fiabilidad de la escala, obteniendo resultados positivos (alpha superior a 0,80 ).
} 
Tabla 5.

Calidad de vida y percepción del cambio social.

Asociaciones entre categorías y porcentaje de cada tipo sobre el total de la muestra.

\begin{tabular}{lccccc}
\hline $\begin{array}{l}\text { Tipos calidad } \\
\text { de vida }\end{array}$ & $\begin{array}{c}\text { Movilidad } \\
\text { social }\end{array}$ & $\begin{array}{c}\text { Percepción cambio } \\
\text { desigualdades } \\
\text { subjetiva }\end{array}$ & $\begin{array}{c}\text { Percepción } \\
\text { oportunidades }\end{array}$ & $\begin{array}{c}\text { Valoración } \\
\text { situación } \\
\text { vitales }\end{array}$ & $\begin{array}{c}\% \\
\text { social }\end{array}$ \\
\hline Bienestar & Descendente & Sociedad Estática & Esperanzada & Igual & 30,5 \\
\hline Disonancia & $\begin{array}{c}\text { Herencia } \\
\text { Descendente }\end{array}$ & Sociedad Riesgo & $\begin{array}{c}\text { Resignada } \\
\text { Pesimista }\end{array}$ & Igual & 11,9 \\
\hline Adaptación & Ascendente & $\begin{array}{c}\text { Sociedad en } \\
\text { progreso }\end{array}$ & Optimista & Mejor & 40,9 \\
\hline Privación & Descendente & Sociedad & $\begin{array}{c}\text { Pesimista } \\
\text { Resignada }\end{array}$ & Peor & 16,7 \\
\hline
\end{tabular}

parte del estado, del tipo que sea (preguntas B1 y B2); la realización o no de labores de autoayuda en el seno de la familia a personas con discapacidades, con problemas económicos, ancianos o acogimiento en el hogar, entre otras posibilidades (pregunta B6). Los resultados del análisis permiten definir tres grandes sectores, además de las prestaciones por desempleo (preguntas B4 y B5):

- Formal: reciben algún tipo de prestación social de carácter específicos (centro de acogida de menores, centro de tercera edad, ayuda económica familiar).

- Familiar: cuando la familia cuida en su propio hogar a personas con problemas (minusválido, anciano, personas con problemas económicos, menores).

- Externo: familias que están fuera de los sistemas anteriores, ni reciben prestaciones sociales específicas, ni realizan labores de cuidado en el hogar.

- Prestaciones por desempleo: tiene en cuenta si una o más personas en la familia reciben prestación económica por encontrarse desempleado.

Los índices relativos a la cultura política en Andalucía muestran una sociedad que se siente poco competente en relación a los asuntos públicos y que muestra un apoyo positivo, aunque moderado, al Estado del Bienestar. El índice de com- 
petencia política muestra que algo más de la mitad de los entrevistados, el 55\%, se sienten incapaces de influir sobre las decisiones del gobierno e incapaces de entender los asuntos públicos; el $29 \%$ se siente capacitado en uno de los dos aspectos, mientras que el $15 \%$ se siente competente en ambos.

Por otra parte, la media de la escala de apoyo al estado de bienestar se sitúa en su punto medio $(3,29)$, y con una distribución asimétrica en la que tienen mayor peso los valores superiores. Este apoyo difuso se muestra al recodificar la escala, pues el porcentaje que apoya poco o muy poco esta forma de gobierno (valores iguales o inferiores a la media) acumulan alrededor de $40 \%$ de los entrevistados.

En lo que se refiere a la relación efectiva que mantienen la población entrevistada con las políticas de bienestar, se evidencia que el 10\% de los hogares reciben prestaciones sociales específicas, es decir, se beneficia de ayudas adicionales a servicios básicos de educación y de salud. El 13\% realiza en el propio hogar labores de cuidado de personas con problemas o ayudan a otras personas fuera de él. El 76\% restante, ni recibe prestaciones sociales específicas ni expresa estar realizando labores de cuidado o prestando ayuda a otras personas. En este sentido, decimos que se mantiene externo al sistema de bienestar, excluidas como se ha dicho la salud o la sanidad. A ello debe sumarse que en alrededor del $12 \%$ de los hogares existe al menos un individuo que percibe prestación económica por desempleo.

La relación existente entre las variables relativas a la cultura política, al sistema de bienestar en Andalucía y a los tipos de bienestar, se muestran en la ilustración 5. En ella puede apreciarse que el primer eje diferencia entre los incluidos en la red formal y los externos al sistema de bienestar, tal como se definieron antes. Los primeros presentan mayor nivel de apoyo al Estado de Bienestar. En el segundo eje diferencia las familias que reciben prestaciones por desempleo de las que realizan labores de cuidado en el hogar (categoría "familiar"). En conjunto, el plano muestra que, como cabría esperar, la externalidad al sistema de bienestar aparece ligada a la situación de bienestar, a un bajo apoyo al estado de bienestar y altos índice de competencia política. El apoyo aumenta y la competencia disminuye a medida que es mayor la inclusión en la red.

Más concretamente, en las situaciones de privación las relaciones con el sistema de bienestar se articulan a través de la red formal y la autoayuda familiar. Las situaciones de adaptación aparecen ligada, sobre todo, a las prestaciones por desempleo. En cambio, las situaciones objetivas favorables presentan niveles de competencia política más altos y menor cercanía y apoyo al estado de bienestar. Pero en el caso de quienes se sitúan en la situación de disonancia el rechazo al estado de bienestar se atenúa y presentan cierto acceso a la red formal y a las prestaciones por desempleo. 
Ilustración 5.

Calidad de vida, cultura política y sistema de bienestar en Andalucía. Saturaciones de las categorías en el primer plano factorial

$(33,60 \%$ varianza $)$.

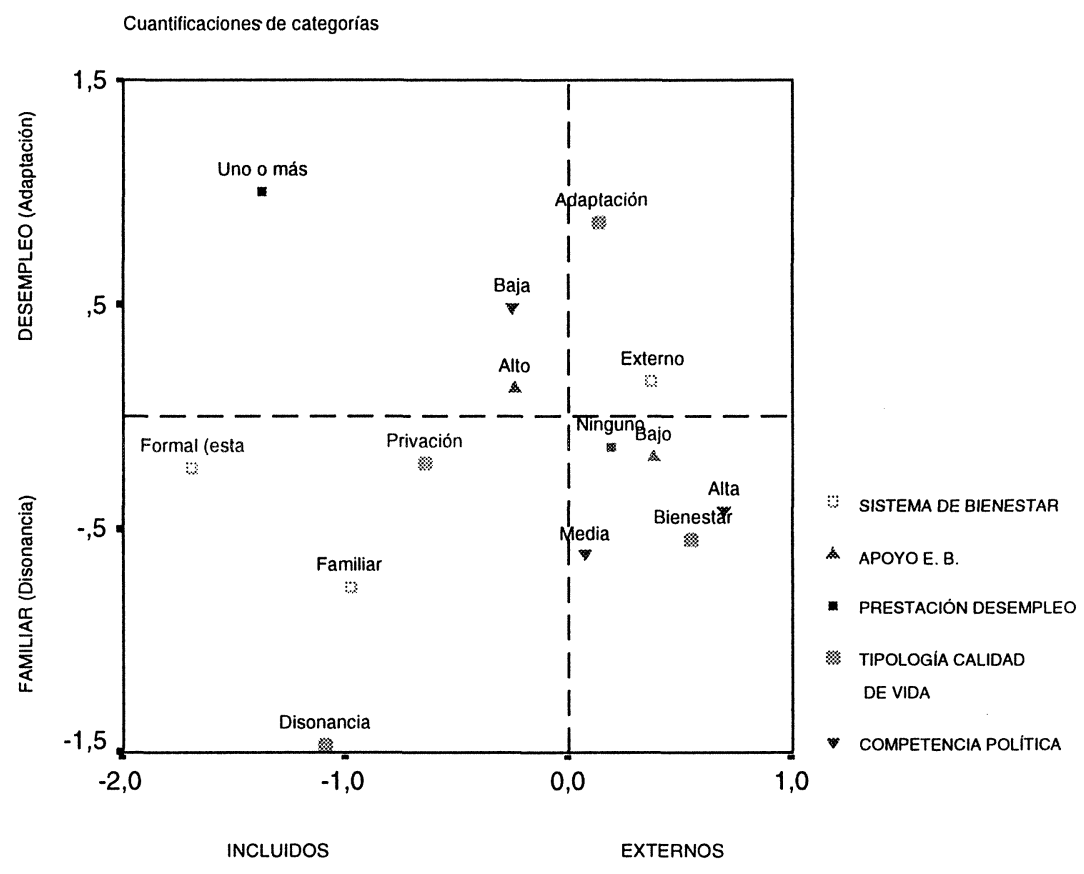

Percepciones, estado y situación objetiva

En este apartado se van a tratar de delimitar de forma más clara los rasgos que definen los cuatro tipos de calidad de vida que venimos utilizando. Para ello se utilizarán las variables más relevantes de los análisis anteriores y tres variables adicionales de carácter socioestrucutural, que son las siguientes:

a) Clase social del cabeza de familia, construida a partir de la ocupación. Resumiendo la propuesta de Golthorpe, se han elaborado las siguientes categorías: clase media, trabajadores no manuales, trabajadores manuales cualificados, trabajadores manuales no cualificados. A estas se han añadido las situaciones de jubilado y parado (o desempleado) ${ }^{16}$.

b) Tamaño de hábitat, medido en número de habitantes: menos de 5.000 , de 5.001 a 20.000 , de 20.001 a 100.00 , y más de $100.000^{17}$. 
Tabla 6.

Calidad de vida, cultura politica y sistema de bienestar en Andalucía. Relaciones entre categorías.

\begin{tabular}{l|c|c|c|c}
\hline $\begin{array}{l}\text { Tipos de } \\
\text { calidad } \\
\text { de vida }\end{array}$ & $\begin{array}{c}\text { Competencia } \\
\text { Política }\end{array}$ & $\begin{array}{c}\text { Apoyo } \\
\text { Estado } \\
\text { Bienestar }\end{array}$ & $\begin{array}{r}\text { Sistema de } \\
\text { Bienestar }\end{array}$ & $\begin{array}{r}\text { Prestación } \\
\text { Desempleo }\end{array}$ \\
\hline Bienestar & Alta & Bajo & Externo & No \\
Disonancia & Media & Medio & Formal & Si \\
Adaptación & Baja & Medio & Externo & Familiar \\
Privación & Baja & Alto & Formal & No \\
\hline
\end{tabular}

c) Nivel de renta, medido a través de la renta media familiar por persona, aplicando la escala de equivalencia OCDE-Oxford. De acuerdo con la distribución de la variable, se han delimitado las siguientes categorías: pobreza (menos de la mitad de la renta media, que para Andalucía está en torno a las 63.000 pesetas mensuales), hasta la media, hasta 95.000 pesetas y más de 95.000 pesetas.

Los resultados a los que se ha podido llegar mediante el análisis de correspondencia entre las variables anteriores parecen mostrar una estructura de asociaciones entre ellas, que permite dar cuenta del perfil social de cada uno de los tipos de calidad de vida delimitados. La ilustración 6 , que refleja el primer plano factorial o estructural de asociación, ordena en el eje horizontal a los entrevistados según su situación objetiva en las variables renta, clase social del cabeza familia y hábitat, cuyos valores positivos se sitúan a la derecha y los negativos a la izquierda del plano. El eje vertical los ordena según cultura política y estructura de oportunidades - experiencias de movilidad, relación con el sistema de

\footnotetext{
${ }^{16}$ Mediante análisis multivariable hemos podido determinar que esta variable, junto a la renta, son las que en mayor medida dan cuenta del estatus social de los entrevistados y de los hogares en su conjunto. Los resultados de este análisis no se incluyen en este texto por razones de espacio. Para su elaboracón se han tomado las indicaciones del Proyecto Internacioncl sobre Estructura, Biografía y Conciencia de Clase. Para más detalle se remite a Carabaña el al. (1992) y González (1992).

${ }^{17}$ En otros análisis sobre la sociedad andaluza, el hábitat se ha mostrado como un indicador relevante en relación a la estructura de oportunidades de acceso a ese proceso. Cif. Del Pino y Bericat (1998), Moyano y Navarro (1998) o Navarro (2000).
} 
bienestar y hábitat-. Las opiniones positivas junto al hábitat rural se sitúan en la parte superior del eje y las negativas y hábitats superiores en la zona inferior. En medio de estos ejes se distribuyen los tipos de calidad de vida.

El tipo "bienestar" aparece como un fenómeno urbano, de clase media con niveles de renta siempre superiores a la media, en un punto neutro en cuanto a la percepción del cambio social (la zona derecha de la ilustración 6). También aparece como urbano el fenómeno de la disonancia y la pobreza, ligadas a percepciones negativas del cambio social, aunque la primera asociada a la clase trabajadora, y la segunda a la situación de desempleado. La adaptación, en cambio, es un fenómeno fundamentalmente rural, propio de jubilados y trabajadores no cualificados.

El tercer eje, representado junto al primero en la ilustración 7, permite concretar las relaciones que tienen entre sí las categorías de las variables. La adaptación, fenómeno rural, aparece en este caso ligada a situaciones de pobreza económica, parados y trabajadores no cualificados. La disonancia, en hábitats mayores, aparece ligada a las mismas clases sociales, pero sin los niveles de pobreza del tipo anterior (zona inferior izquierda de la ilustración). Por último, la privación se asocia a ciudades de tamaño intermedio, entre 20.000 a 100.00 habitantes, y a la condición de jubilado (zona superior izquierda de la ilustración).

En su conjunto, los resultados del análisis permiten realizar la caracterización tipológica que aparece en la tabla 7, en donde cada uno de los tipos deben tomarse más como tipos ideales, más en forma de tendencias sociales, que como descripción exhaustiva.

\section{CONCLUSIONES}

Los análisis anteriores muestran que la población que presenta la paradoja de la satisfacción se caracteriza por situaciones de privación, aunque integrada en el sistema de bienestar. Se trata de trabajadores no cualificados del ámbito rural que, a pesar de su escasa o discontinua inserción laboral, cuentan con el apoyo de las prestaciones por desempleo, o de jubilados que acceden, por medio de sus pensiones, a niveles de renta que los alejan de la situación de pobreza. Pero ambos, en un marco social en el que el acceso a la vivienda, aún presentando siendo difícil, no lo es tanto como en el ámbito urbano. Además, a las tradicionales redes de bienestar, propias del ámbito rural, se une la acción del Estado de Bienestar, abriendo una nueva y amplia estructura de oportunidades de inserción social. Todo ello da lugar a una percepción muy positiva tanto del cambio social, como de las perspectivas de futuro. En fin, la población parece vivir "a gusto" en una situación que, aún teniendo todavía carencias y necesidades, era poco imaginable una o dos generaciones antes. La satisfacción se debe, pues, a la comparación de las mejoras habidas con respecto a la situación en el pasado. 
Ilustración 6

Saturaciones de las categorías en el primer plano factorial $(46,13 \%$ varianza).

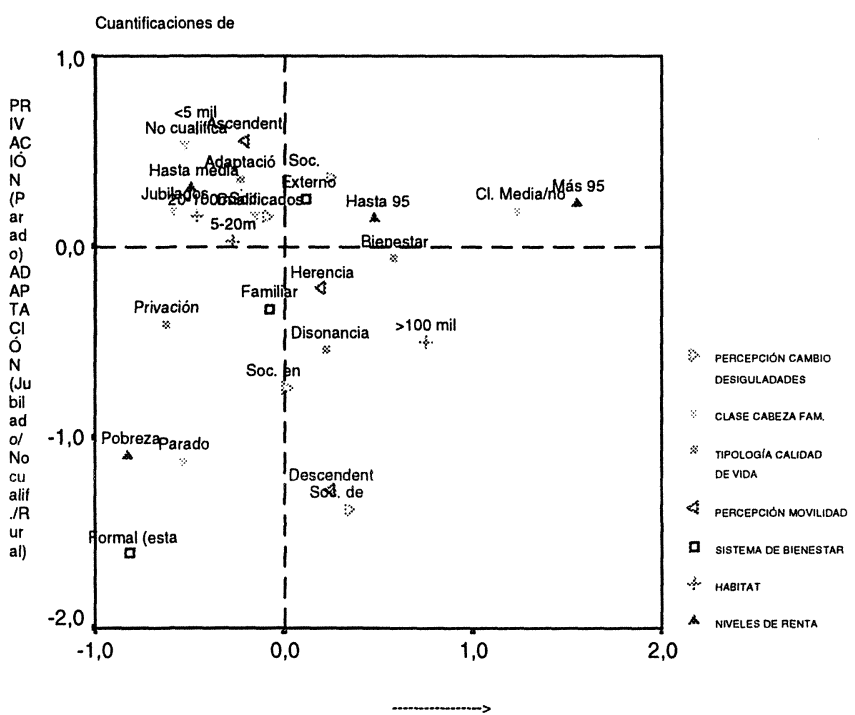

Ilustración 7.

Saturaciones de las categorías en el primer plano factorial $(32,33 \%$ varianza $)$.

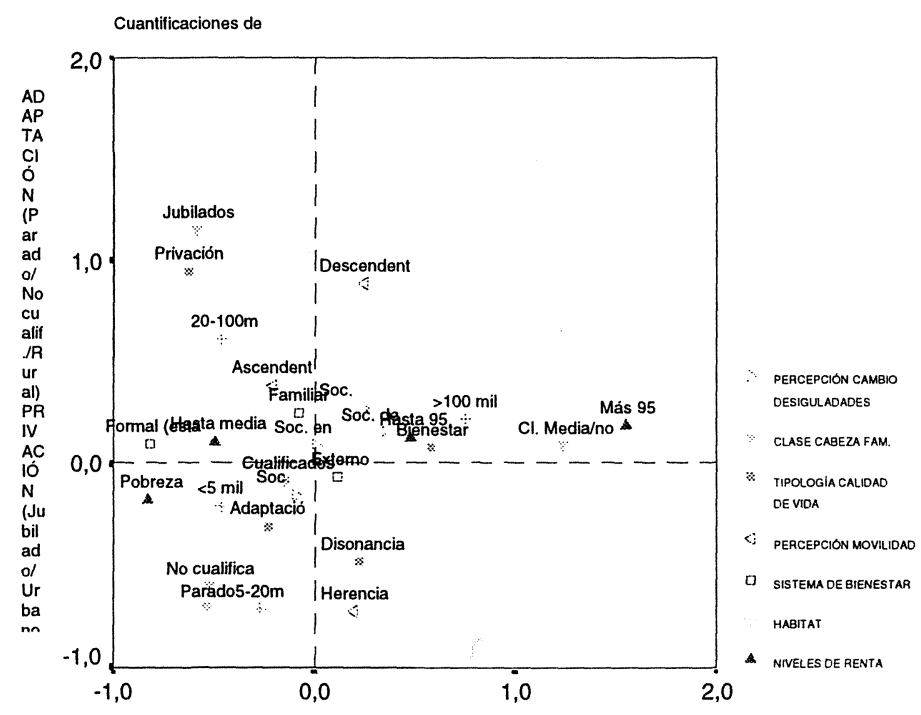


Tabla 7.

Caracterización tipológica de los tipos de calidad de vida en Andalucía.

\begin{tabular}{|c|c|c|c|c|c|c|}
\hline Tipos & $\begin{array}{l}\text { Posición } \\
\text { social } \\
\text { cabeza } \\
\text { familia }\end{array}$ & $\begin{array}{c}\text { Renta } \\
\text { familiar } \\
\text { por } \\
\text { persona }\end{array}$ & Habitat & $\begin{array}{l}\text { Acceso } \\
\text { red formal } \\
\text { sistema } \\
\text { bienestar }\end{array}$ & $\begin{array}{c}\text { Acceso a } \\
\text { prestación } \\
\text { desempleo }\end{array}$ & $\begin{array}{l}\text { Percepción } \\
\text { cambio } \\
\text { social }\end{array}$ \\
\hline \multirow[t]{2}{*}{ Privación } & Parados & Pobreza & Urbano & Alto & Bajo & \\
\hline & Jubilados & $\begin{array}{c}\text { Menos media } \\
\text { y Probreza }\end{array}$ & Intermedio & Alto & No & Negativa \\
\hline \multirow[t]{2}{*}{ Adaptación } & Jubilados & $\begin{array}{c}\text { Entre pobreza } \\
\text { y media }\end{array}$ & Rural & Bajo & No & Muy positiva \\
\hline & $\begin{array}{c}\text { Trabajadores no } \\
\text { cualificados } \\
\text { (Parados) }\end{array}$ & & & & Alto & \\
\hline Disonancia & $\begin{array}{c}\text { Trabajadores } \\
\text { no cualificados } \\
\text { (T.cualificados) }\end{array}$ & $\begin{array}{c}\text { Entre media } \\
\text { y } 95.000\end{array}$ & Urbano & Alto & Bajo & $\begin{array}{l}\text { Más bien } \\
\text { negativa }\end{array}$ \\
\hline \multirow[t]{2}{*}{ Bienestar } & $\begin{array}{l}\text { Trabajadores } \\
\text { no manuales }\end{array}$ & $\begin{array}{c}\text { Entre media y } \\
95.000\end{array}$ & \multirow[t]{2}{*}{ Urbano } & \multirow[t]{2}{*}{ No } & \multirow[t]{2}{*}{ No } & \multirow[t]{2}{*}{ Neutra } \\
\hline & Clase media & Más 95.000 & & & & \\
\hline
\end{tabular}

Ahora bien, todo ello tiene lugar en un marco cultural tradicional, caracterizado por el sentimiento de incompetencia política, de que el cambio depende más de fuerzas externas que de la propia acción. Rasgos de una cultura política más cercana al modelo de ciudadanos que depositan altas expectativas en la intervención estatal para la solución de los problemas que al de ciudadanos activos involucrados directamente en su solución. Existe, pues, apoyo al Estado de Bienestar, a su resultados, pero desde niveles bajos de competencia política, ciudadanos que se sienten incapaces para actuar e informarse en relación a los asuntos públicos. Esto puede reforzar el mismo modelo de cultura política y, además, mantener constantes e incluso incrementar las demandas al estado sin una contrapartida de participación y compromiso cívico activo.

En hábitats de mayor tamaño se sitúa otro grupo de ciudadanos y ciudadanas políticamente incompetentes que, compartiendo ciertas características con el anterior, se sitúan en una situación de privación. Se trata de hogares donde el cabeza de familia está desempleado, al igual que otros miembros, con niveles de 
renta que los colocan en situación de pobreza o cercanos a ella que, apoyados fundamentalmente en la autoayuda familiar, acceden en menor medida que los anteriores a prestaciones sociales específicas, y sobre todo, a las de desempleo. A ello se añade la disolución del apoyo de una red difusa de sociabilidad atenta a las carencias que sufren. Este rasgo, ligado a las diferencias entre hábitats de distinto tamaño, se muestra revelador para el caso de los jubilados de zonas urbanas, pues aún mostrando un perfil similar al de los jubilados rurales, se sienten menos satisfechos con su vida.

Así pues, a diferencia de la situación de adaptación, en la de privación se han sentido y sienten las externalidades del proceso de modernización, en la formas de desempleo o desintegración social. Esto se percibe desde un sentimiento general de impotencia, de incapacidad de conocer e influir sobre los asuntos públicos. Todo ello se manifiesta en una percepción negativa y pesimista del cambio social y las posibilidades de mejora en el futuro.

En la situación de disonancia la población presenta rasgos similares, sobre todo en lo que se refiere a la dimensión moral del proceso de modernización, pues objetivamente su situación es más favorable. Se trata, fundamentalmente, de la clase obrera urbana, trabajadores cualificados, pero sobre todo no cualificados, en hogares donde suelen existir experiencias de desempleo, con rentas por encima de la media, pero por debajo de las 95.000 pesetas. Recurren a la autoayuda familiar, pero acceden en menor grado que los tipos anteriores a las prestaciones por desempleo. En suma, una situación favorable pero de riesgo, percibida desde un sentimiento general de impotencia e incompetencia política, ligada a una visión resignada del cambio social y, sobre todo, de las perspectivas de futuro.

Frente al optimismo de los adaptados, la impotencia de los que se encuentran en situación de privación y la resignación de los insatisfechos, la clase media urbana se sitúan en los mejores niveles de condiciones de vida y con niveles altos de satisfacción. Ciudadanos más competentes políticamente que los grupos anteriores y más orientados a valores propios de la sociedad moderna (logro personal, individuación, autonomía), que prestan un apoyo menor al Estado de Bienestar, y con una visión del cambio social más bien neutra. Una población satisfecha, pero, sobre todo, satisfecha consigo misma más que con los cambios que se han producido en la sociedad andaluza. No dejan de reconocer el cambio positivo habido en ésta, pero es previsible que este grupo perciba que las mejoras se deben a su esfuerzo personal, al logro individual, más que a la acción pública.

A lo largo de este trabajo se ha puesto reiteradamente de manifiesto que el proceso de modernización se ha hecho cada vez más evidente en la sociedad andaluza, pero sobre todo en su dimensión material, habiendo quedado rezagada su dimensión cultural o axiológica. Se trata, pues, de una sociedad modernizada aunque no del todo, por que existen pautas culturales tradicionales que aún tienen un peso apreciable frente a las modernas. Los resultados del análisis y su 
Tabla 8.

El proceso de modernización, la percepción del cambio social y la calidad de vida en Andalucía.

\begin{tabular}{|c|c|c|c|c|c|c|c|}
\hline \multirow{3}{*}{$\begin{array}{l}\text { TIPOLOGÍA } \\
\text { DE CALIDAD } \\
\text { DE VIDA }\end{array}$} & \multicolumn{5}{|c|}{ CAMBIO SOCIAL } & \multicolumn{2}{|c|}{$\begin{array}{l}\text { PERCEPCIÓN DEL CAMBIO } \\
\text { SOCIAL }\end{array}$} \\
\hline & \multicolumn{2}{|c|}{$\begin{array}{l}\text { MODERNIZACIÓN: } \\
\text { DIMENSIÓN MATERIAL }\end{array}$} & \multicolumn{3}{|c|}{$\begin{array}{l}\text { MODERNIDAD: } \\
\text { DIMENSIÓN MORAL }\end{array}$} & & \\
\hline & $\begin{array}{c}\text { Externalidades: } \\
\text { pobreza y } \\
\text { desempleo }\end{array}$ & $\begin{array}{l}\text { Acceso al } \\
\text { estado } \\
\text { benefactor }\end{array}$ & Individuación & $\begin{array}{c}\text { Integración } \\
\text { social } \\
\text { y ayuda } \\
\text { mutua }\end{array}$ & $\begin{array}{l}\text { Cultura } \\
\text { política }\end{array}$ & Pasado & Futuro \\
\hline PRIVACIÓN & ++ & + & \multirow[b]{2}{*}{-} & + & \multirow{2}{*}{$\begin{array}{l}\text { Incompetente } \\
\text { y estatalista }\end{array}$} & Muy negativa & Impotencia \\
\hline ADAPTACIÓN & & ++ & & ++ & & Muy positiva & Optimismo \\
\hline DISONANCIA & + & + & & - & & Negativa & Resignación \\
\hline BIENESTAR & - & - & + & - & Competente & Neutra & Tranquilidad \\
\hline
\end{tabular}

Los signos indican la presencia (+) o ausencia (-) de los rasgos para cada tipo. 
discusión permiten afirmar que este desajuste puede dar cuenta de las distintas situaciones de calidad de vida. Pero, sobre todo, de la importancia relativa del proceso de adaptación y de la aparente paradoja de la sociedad satisfecha. En suma, frente a los incluidos en el proceso de modernización (bienestar) y los excluidos de él (privación), aparecen situaciones intermedias que pueden llegar a explicarse por el desajuste entre las dimensiones cultural y material de este proceso. De forma resumida esto se refleja en la tabla 8.

Lo expuesto da pie para pensar que el proceso de modernización presenta riesgos y efectos no queridos para la sociedad andaluza. Riesgos evidentes por lo que se refiere a los excluidos de él, pero también para aquéllos que aun encontrándose en situaciones más favorables, no terminan de incorporarse al cambio cultural que implica la modernización, manteniendo pautas poco adecuadas para intentar mejorar su situación de riesgo, pues se orientan hacia el estado como mecanismo exclusivo de dinamización social.

Los efectos no queridos cabe localizarlos entre ese amplio, e incluso mayoritario grupo, que han ido progresivamente adaptando de forma realista sus aspiraciones a su situación. Ahora bien, esa adaptación parece provenir de actitudes y valores premodernos, en donde la ligazón al proceso de modernización es inducida externamente por la acción pública, sin que se desarrollen esquemas axiológicos o iniciativas orientadas al esfuerzo y al logro personal. El sentimiento es de satisfacción, pero sobre todo porque se ha mejorado con cierta independencia de la acción individual.

En suma, puede argumentarse que la extensión del estado del bienestar ha logrado mejorar las condiciones de vida de la población andaluza, lo que se refleja en altos niveles de satisfacción. Pero también que esto esconde, e incluso pudiera potenciar, el mantenimiento de esquemas axiológicos tradicionales y poco proclives al dinamismo y a la iniciativa propias de la modernidad. O aún más, la creación de una sociedad polarizada entre un segmento de ciudadanos con carencias y necesidades pero satisfechos, y otro en situación de bienestar, satisfechos con ellos mismos más que con la acción pública del Estado de Bienestar y que con la sociedad en la que viven. En suma, una reducción de la polarización social por el lado de las condiciones de vida, pero la creación de una sociedad civil axiológicamente polarizada.

\section{BIBLIOGRAFÍA}

ABBEY, A. y F.M. ANDREWS (1985), "Modeling the psychological determinants of life quality", en Social Indicators Research, no 16, pp. 1-34.

ABRAMSON, L.Y., M.E. SELIGMAN y J.D. TEASDALE(1978), "Learned helplessness in humans: Critique and reformulation", Journal of Abnormal Psychology, $\mathrm{n}^{\circ}$ 87, pp. 49-74. 
AGUIAR, F. et al. (1995), Indicadores sociales de calidad de vida y tercera edad, Documento de Trabajo del IESA/CSIC (DT 95-05), Madrid, IESA/CSIC.

ALLART, E. (1978), "Objetive and subjetive social indicators of well-being", Comparative Studies in Sociology, $\mathrm{n}^{\circ}$ 1, pp. 142-173.

ALMOND, G. y S. VERBA (1963), La Cultura Cívica, Madrid, Euroamérica.

ANDREWS, F. y S. WHITE (1976), Social Indicators of Well-being in America, Nueva York, Plenum.

ANDREWS, F.M. y J.P. ROBINSON (1991), "Measures of Subjetive Well-being", en Robinson, J.P., Ph.R. Shaver y L.S. Wrightsman (eds.), Measures of Personality and Social Psychological Attitudes, San Diego, Academic Press, pp. 61-114.

BARNES, S.H. y M. KAASE (eds.) (1979), Political Action: mass participation in five western democracies, Beverly Hills, Sage.

CAMPBELL, A., Ph. E. CONVERSE y W.L. ROGERS (1976), The Quality of Life, Nueva York, Russell Sage.

CARBAÑA, J., J.J. GONZÁLEZ, C.J. NAVARRO, A. SERRANO, A. VALLEJO y S. VEREDAS (1992), Encuesta de Estructura, Biografía y Conciencia de Clase. Descripción detallada del proyecto, definiciones, depuración de la información y ficheros de datos, Documento de Trabajo, Consejería de Economía/Comunidad de Madrid.

CHAMBERLAIN, K. y S. ZIKA (1992), "Stability and change in subjetive well-being over short time periods", Social Indicators Research, n² 26, pp. 101-117.

C.E.S. (1996), La pobreza y la exclusión social en España, Serie Informe $\mathrm{n}^{\circ}$ 8, Madrid, C.E.S.

DEL PINO, J. y E. BERICAT (1998), Valores sociales en la cultura andaluza, Madrid, CIS.

DELEECK, H. y K. VAN DEN BOSCH (1990), "Dimensión de la pobreza en un contexto comparativo: evidencia empírica y evaluación motodológica de cuatro umbrales de poreza en siete países de la Comunidad Europea", Cuadernos de Acción Social, n² 24, pp.76-107.

DIENER, E. (1994), "Assesing subjetive well-being: progress and opportunitties", en Social Indicators Research, $\mathrm{n}^{\circ} 31$, pp. 103-157.

DIENER, E., E. SANDVIK, L. SEIDLITZ y M. DIENER (1993), "The relationship between income and subjetive well-being: relative or absolute?", Social Indicators Research, $\mathrm{n}^{\circ} 28, \mathrm{pp} .195-$ 223.

DICKENS, P. (1990), Sociologia Urbana, Bologna, il Mulino.

DOYAL, L. y I. GOUGH (1991), A Theory of Human Need, Londres, McMillam.

ELSTER, J. (1979), Ulysses and the Sirens, Cambridge, Cambridge University Press. 
(1983), Sour Grapes, Cambridge, Cambridge University Press.

FERNÁNDEZ ESQUINAS, M. et al. (1998), Encuesta sobre Calidad de vida en Andalucía. Diseño muestral y organización del trabajo de campo. Córdoba, IESA-A, Documento de Trabajo DT98-7.

FESTINGER, L. (1978), Theory of Cognitive Dissonance, Huber, Suttgar.

FLETCHER, C.N. y F.O. LORENZ(1985), "Structural influences on the relationship between objetive and subjetive indicators of economic well-being", Social Indicators Research, $\mathrm{n}^{\circ} 16, \mathrm{pp} .333$ 345 .

GONZÁLEZ, J.J. (1992), "La construcción empírica de las clases", Política y Sociedad, n 11, pp. 99-122.

HORLEY, J. y J.J. LAVERY (1991), "The stability and sensitivity of subjetive well-being measures", Social Indicators Research, no 24, 113-122.

INGLEHART, R. (1991), El cambio cultural en las sociedades induatriales avanzadas, Madrid, CIS.

IPSEN, D. (1978), "Das Konstrukt der Zufriedenheit”, en Soziale Welt, n² 29, pp. 44-53.

KYMBLICA, N. y N. NORMAN (1994), "Return of the Citizen: A Survey of Recent Work on Citizenship Theory", Ethics, n 104, pp. 352-381.

MARTINSON, O.B., E.A. WILKENING y D. McGRANAHAN (1984), "Predicting overall life satisfaction: the role of life cycle and social psychological antecedents", Social Indicators research, $\mathrm{n}^{\circ} 14$, pp. $99-108$.

MICHALlOS, A.C. (1980), North America Social Report, Dordrecht, D. Reidel.

MOYANO, E. y C.J. NAVARRO (1999), El movimiento de consumidores en Andalucía, Sevilla, Consejería de Economía de la Junta de Andalucía.

MOYANO, E. y M. PÉREZ YRUELA (coords.) (1999), Informe Social de Andalucía (1977-97). Dos décadas de cambio social,Córdoba, IESA-Publicaciones del CSIC.

NAVARRO, C.J. (1988), El Nuevo Localismo. Municipio y democracia en la sociedad global, Córdoba, Servicio Publicaciones Diputación de Córdoba.

(2000), Cultura política, ambientalismo y cambio social, Sevilla, Aconcagua Editores.

OLSON, G.I. y B.I. SCHOBER (1993), "The Satisfied Poor", Social Indicators Research, nº 28, pp. 173-193.

PÉREZ-DÍAZ, V., E. CHULIÁ y B. ALVAREZ MIRANDA (1998), Familia y sistema de bienestar, Madrid, Visor. 
YETIM, U. (1993), "Life satisfaction: A study based on the organization of personal projects", en Social Indicators Research, n 29, pp. 277-289.

SUMMARY. Results derived from an empirical research about life conditions in andalusian society are presented in this article. The research is based on an opinion survey carried out to a sample of 2.400 cases. The research has been designed taking into account the contemporary trend of analysing at the same time the objectives changes occurred in the objective life conditions in the past years and the subjective opinion citizens have about these changes. Comparing the objective and subjective dimensions, a typology has been developed according to the position people have in both aspects. The work focus in the type called "adaptation", that include those cases in which objective life condition are worst than the average of the total population, but nevertheless people declare to be satisfied with this situation. A hypothesis is discussed in order to explain this paradox, being the explanation that satisfaction in the type "adaptation" is due to an optimistic evaluation by those included in it of both past and expected social change.

E-mail: cnavyan@dts.upo.es mpyruela@iesaa.csic.es 